\title{
From Sensing to Intervention for Mental and Behavioral Health
}

\author{
Varun Mishra \\ Dartmouth College \\ varun@cs.dartmouth.edu
}

\section{CCS CONCEPTS}

- Human-centered computing $\rightarrow$ Ubiquitous and mobile computing; • Applied computing $\rightarrow$ Health care information systems;

\section{KEYWORDS}

Receptivity; intervention delivery; stress detection; mental health; mobile health (mHealth); engagement

\section{ACM Reference Format:}

Varun Mishra. 2019. From Sensing to Intervention for Mental and Behavioral Health. In Adjunct Proceedings of the 2019 ACM International foint Conference on Pervasive and Ubiquitous Computing and the 2019 International Symposium on Wearable Computers (UbiComp/ISWC '19 Adjunct), September 9-13, 2019, London, United Kingdom. ACM, New York, NY, USA, 5 pages. https://doi.org/10.1145/ 3341162.3349304

\section{INTRODUCTION AND PROBLEM STATEMENT}

Advances in mobile, wearable and embedded sensing technology have created new opportunities for research into a variety of health conditions. This has led to the field of mobile health (mHealth), which covers a full spectrum of works, including but not limited to disease surveillance, treatment support, epidemic outbreak tracking, and chronic disease management. An important sub-field which has been rising in the past years is the application of mHealth technology in the field of mental and behavioral health, enabling researchers to study stress, depression, mood, personality change, schizophrenia, physical activity and addictive behavior, among other things.

The ubiquitous presence of sensor-rich mobile and wearable devices has helped researchers to passively track and

Permission to make digital or hard copies of all or part of this work for personal or classroom use is granted without fee provided that copies are not made or distributed for profit or commercial advantage and that copies bear this notice and the full citation on the first page. Copyrights for components of this work owned by others than the author(s) must be honored. Abstracting with credit is permitted. To copy otherwise, or republish, to post on servers or to redistribute to lists, requires prior specific permission and/or a fee. Request permissions from permissions@acm.org. UbiComp/ISWC '19 Adjunct, September 9-13, 2019, London, United Kingdom (c) 2019 Copyright held by the owner/author(s). Publication rights licensed to ACM.

ACM ISBN 978-1-4503-6869-8/19/09...\$15.00

https://doi.org/10.1145/3341162.3349304 sense user characteristics, e.g., location, activity, device interaction, sociability, and even physiological signals, and build machine learning models to, among others, detect or predict mental or behavioral health conditions, with varying degree of accuracy. The eventual goal is to accurately detect and provide appropriate support or intervention to cope with the respective condition. While a lot of work has been done in this domain, there exist several challenges before meaningful interventions can be provided to users.

Our work explores and advances the complete "lifecycle" of mental and behavioral health research - from sensing to intervention. We envision there are three major components in this lifecycle: (a) accurate sensing and detection of the mental or behavioral health condition, e.g., stress, anxiety, substance use; (b) determining when is the right time to deliver the intervention, i.e., when a user is most likely to be receptive to the provided support (state-of-receptivity); and (c) determining the best intervention delivery mechanism in that moment, i.e., the choice of device for delivering that intervention based on the context of the person to ensure just-in-time delivery and reachability; this mechanism could be a notification on the phone or a sound or display on an ambient smart-home device. Simply put, the three components loosely represent the "what", "when" and "how" for mental and behavioral health research. We show a visual representation of this lifecycle with the respective labels in Figure 1. It is, however, important to note that the transition from $(a)$ to $(b)$ is contingent on the choice of appropriate intervention or support as determined by psychologists and clinicians. Design and choice of interventions is beyond the scope of this work.

In the past, researchers have explored a variety of mental and behavioral health conditions. In our work, we explore, advance and contribute to the different components of the "lifecycle" for specific health outcomes, with the eventual goal to provide a foundation for an end-to-end cycle that can hopefully be adapted to or be useful for different health conditions. For the sensing and detection component $(a)$, we focus on mental stress, and for the intervention components ( $b$ and $c$ ), we focus on JITAIs (Just-in-time Adaptive Interventions) to improve physical activity behavior.

Ideally, a perfect "lifecycle" would be to detect and provide interventions for the same health outcome, e.g., stress; 


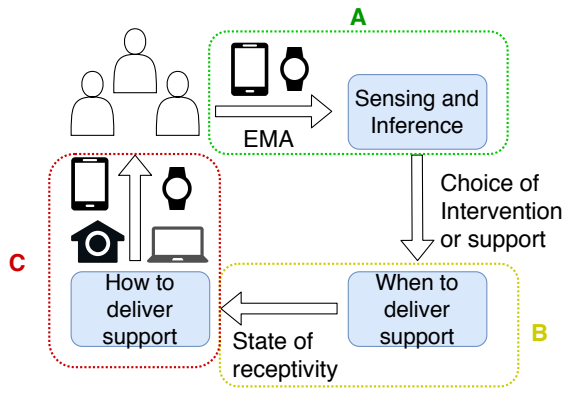

Figure 1: The sensing to intervention lifecycle for mental and behavioral health research.

however, we found that prior work in stress (and most mental health conditions) detection in-the-wild, i.e., in daily living conditions, was not accurate enough (or generalizable enough) to provide meaningful interventions. Hence, to be able to tackle challenges in different components in parallel, we chose to explore receptivity towards physical activity interventions as there exists an empirical measurement (step count) for physical activity levels; something that can be measured accurately using a smartphone. The interventions (and metrics for receptivity) were designed such that the factors for receptivity could be applicable to similarly designed interventions for other health conditions.

In this paper, we discuss the background, our methodology, research progress and (expected) contributions in each individual component: (1) stress detection, (2) state-of-receptivity towards JITAI, and (3) modalitiy for intervention delivery.

\section{BACKGROUND AND RELATED WORK}

\section{Stress Detection}

The sustained experience of stress may lead to emotional concerns (such as anger, anxiety, or acute periods of depression), and physical distress (such as headaches, digestive problems, and even diabetes) [18]. Chronic stress can have a domino effect on other mental and behavioral outcomes. Hence, we identify stress as a fundamental condition, proper management of which can lead to an improvement in other mental and behavioral health conditions, e.g., smoking cessation, depression, and drug use.

Improvements in sensors and sensing capabilities over the years have led to a spectrum of prior work in stress detection and assessment in a variety of situations (a) stress induced in a lab, where researchers ask the participants to undergo some well-validated stress-inducing tasks $[1,3,5]$, (b) constrained real-life situations, where the researchers monitored the user's stress level when they were in a particular situation, e.g., while driving [4], or while sleeping [13], and (c) in unconstrained free-living situations $[3,5]$; using a variety of sensors, e.g., Electrocardiography (ECG) [3, 5], Galvanic Skin Response (GSR) sensor [1, 3, 4], Blood Volume Pulse (BVP) sensor [3], or Electromyogram (EMG) sensor [4].

The prior work in stress detection, however, has several limitations which have hindered large-scale adoption and validation of methods or results. In several of these works, the researchers have built their custom-made sensing system, or use expensive clinical-grade hardware, which leads to a lack of reproducibility by other researchers and lack of large-scale deployments with different user groups. Further, the variance in devices, sensors, and methodologies used by different researchers result in the absence of a standardized processing pipeline for the task of stress detection, which can serve as a benchmark.

\section{Receptivity towards Interventions}

Just-in-time adaptive intervention (JITAI) is a novel intervention design, which aims to provide the right type and amount of support, at the right time, adapted as-needed for the individuals' internal and contextual state [14, 15]. JITAIs are most effective if delivered at the right time. But what is the 'right' time? First, they should be delivered at the onset of the relevant behavior or health condition; assuming accurate sensing and detection of the condition, the interventions then should be delivered at a moment when the person will actually see, absorb, and engage with the intervention.

Researchers have explored the related concept of interruptibility, defined as a person's ability to be interrupted by a notification by immediately taking action to open and view the notification content [8]. Prior work has explored the relationship between interruptibility and a variety of factors, like location [12], activity [16], boredom [17], personality [9], phone interaction [17] and more. Although such research may be effective at delivering routine phone notifications, none of those methods are sufficient for timing notifications intended as behavioral interventions. For most phone-based notifications, e.g., messages, email, and social media, the recipient is interruptible if they derive an immediate and clear benefit - either a transfer of important information or the opportunity for an amusing diversion. Behavioral interventions are different. Even participants who are motivated for behavioral change may prefer to ignore or postpone specific intervention activities... particularly if they require challenging behaviors.

Virtually no studies have investigated receptivity for actual mHealth based interventions. Sarker et al. [19] made one such attempt; instead of delivering actual interventions, however, they used surveys and Ecological Momentary Assessment (EMA) questions that they claimed would have similar engagement levels as interventions. The authors also provided monetary incentives to encourage the participants 
to respond to EMA prompts, which might have biased the participants' receptivity.

\section{Modality for Intervention Delivery}

Individuals these days use and interact with more and more devices daily, e.g., smartphone, laptop/computer, tablets, wearables, and smart-home devices. Hence, delivering interventions using just the smartphone might not be the most optimal solution. We hypothesize, given the context, the choice of device will play an essential role in ensuring just-in-time delivery and receptivity of an intervention.

There is limited research in choosing the right device for notification delivery. Mehrotra et al. propose one of the first methods to tackle the problem of delivering notifications on the right device [7]. The authors, however, focus on routine phone notifications, and distinguish the devices as "Mobile" and "Other".

\section{METHODOLOGY}

We conduct our work as part of different multi-disciplinary teams, including psychologists, behavior scientists, and computer scientists. In each of the three components, we have done (or plan to do) a series of experiments and studies. For each component, we identified the challenges that existed in prior work to form our research goals. We next collaborated with other research groups to conduct different studies, as well as to test our models and methods on the data collected by those groups. In some cases (e.g., stress detection), we additionally used publicly available datasets to verify our models further. Our usual research strategy was to start with some exploratory analyses and then discuss novel methods, features, or algorithms to create generalizable solutions. We explain the detailed experiments, studies, and results for each component in the following section.

\section{RESEARCH CONDUCTED AND EVALUATION}

In this section, we discuss the different research questions in each component followed by the research conducted (or planned) along with the results obtained.

\section{Stress Detection}

Based on our analysis of prior work on stress detection, we identified several challenges, which led to the following research questions: (1) can we use a cheap commodity sensor to accurately detect stress?; (2) will methods and approach used for modeling stress using commodity hardware translate to clinical or custom-made devices?; (3) will our methods and models generalize to studies conducted in different locations and population demographics?; (4) can we detect stress for an individual as new data is available in real-time, instead of detecting stress after all of the data for a user is collected?; and (5) can we use contextual information about an user to better detect stress in-the-wild?

To answer questions (1) and (2), we conducted an initial study with 26 participants, recruited from a US university. The study protocol involved a lab session, where the participants went through three validated stress-inducing tests; followed by a 3-day field session, where the participants wore the sensors in their natural environment and were asked to respond to EMAs about their stress levels. The participants wore a commodity heart-rate sensor - Polar H7 - for measuring R-R interval and a custom-made GSR sensor. During analysis, we tried out different data processing methods to find the optimal pipeline for processing physiological signals for the task of stress detection. We further, proposed a novel two-layered stress detection approach, which accounts for the stress probability at a time $t$ to effectively determine the stress probability at a time $t+1$.

Our analysis showed that using the Polar H7 sensor alone, we were able to detect stressful events with an F1 score of up to 0.87 in the lab and 0.66 in the field, on par with clinical-grade sensors. Further, the methods and modeling techniques were applicable to custom-made sensors as well. The heart-rate data from the Polar, when augmented with the GSR sensor, led to an F1 score of 0.94 in the lab, and 0.73 in the field. The preliminary version of this work was published at the MHSI workshop at UbiComp 2018 [11] and the detailed follow-up work is currently under revision at the ACM Transactions on Computing for Healthcare.

To answer questions (3) and (4), we collaborated with other researchers to test our models (built using our initial study) on different datasets. For both these questions, our methods have been tested only on the lab-data, due to the lack of a field-data in most of the studies. We tested our models for generalizability on datasets collected from (i) employees of an IT company in the US, (ii) students of a high school in the US, (iii) students of a university in the US, (iv) a publicly available dataset, WESAD [20]. In total, we evaluated our models with over 120 participants across different studies. Further, we build novel time-series models to detect stress both after the data has been collected for a participant and in real-time as new data comes in for a participant. We are currently preparing to submit this work to IMWUT.

To answer question (5), we did some early work to determine how a person's perception of stress changes with context. To this end, we conducted an 'in-the-wild' study with 23 participants, where we collected physiological data from the users, along with 'high-level' context labels, and perceived stress levels. Our analysis showed that context plays a significant role in the users' perceived stress levels, and when used in conjunction with physiological signals leads to significantly higher stress detection results, as compared to relying on just physiological data [10]. 


\section{Receptivity towards Interventions}

Given the lack of research in understanding receptivity towards actual mHealth interventions, our objective was clear: to conduct a study where participants received actual interventions for a positive behavioral outcome, and explore participants' receptivity towards those interventions. To this end, we created a chat-bot based digital coach, which would deliver interventions for improving physical activity by setting daily step goals. The coach was based on the opensource MobileCoach framework [2], and was available on iOS and Android. We conducted our 6-week study with 189 participants, representative of the German-speaking part of Switzerland. The research protocol for this study has been published at JMIR [6]. We defined various metrics for gauging participants' receptivity towards intervention. We observed several intrinsic factors (i.e., participant-specific characteristics, e.g., age, device type, and personality) and contextual factors (e.g., time, location, activity, device usage) played an important role in shaping participant receptivity. We further observed, participants who were receptive to interventions, were more likely to complete their step goals for the day, and participants who achieved their step goals were more likely to be receptive to interventions the next day. We finally build machine learning models, as a feasibility test, to infer when participants were more likely to be receptive. Even with fairly simple classifiers, we observed significant improvements in F1-score of up to $77 \%$ over a naive classifier baseline. This work is currently under revision at IMWUT.

Current and Proposed Research: Our current work focuses on extracting novel features from the data collected and applying sophisticated methods to build generalized and personalized classifiers for better detecting state-of-receptivity. We further intend to test our models on an independently conducted study, where over a 1000 participants received personality change interventions (this study was also done with a digital coach, and collected similar contextual data). Testing our models on a different study will validate our model and test the generalizability of our receptivity metrics and approach for different behavioral outcomes.

Further, we are planning to conduct a smaller version of the previous physical activity intervention study where the participants will receive interventions based on the machine learning models developed in our prior work. We plan to implement the models in the iOS app, and the app will make real-time decisions on the state-of-receptivity of the user. The model will also have an online learning component, where the model updates itself, in the field, based on the actual receptivity of the user. The goal of this study is to observe how do the different models perform in-the-moment, as compared to delivering interventions randomly.

\section{Modality for Intervention Delivery (proposed)}

We present the third component as proposed work. In this work, we propose to conduct an exploratory data collection study where the participants receive interventions simultaneously on the different devices they possess, e.g., smartphone, laptop, and wearables. Once a participant responds to the message on a particular device, the alert will be removed from the other devices. The goal of this study is to explore if and how the choice of device varies with context. Another question we intend to explore is the overlap between the state-of-receptivity and the device choice, i.e., do there exist situations where the participant is in a state-of-receptivity on one device and not the other? Based on the analyses and results from this study, we hope to build algorithms that can decide on the choice of device for intervention deliver in-the-moment to ensure just-in-time delivery and maximize receptivity towards interventions.

\section{5 (EXPECTED) CONTRIBUTIONS}

In our work, we explore and advance the major components in the lifecycle of mental and behavioral health research. While we make specific contributions in individual components, the overall work lays the foundation for an end-to-end pipeline, starting from sensing to the successful delivery of interventions, by answering the "what", "when" and "how" questions of the lifecycle highlighted in Figure 1.

We list specific contributions (and expected contributions) in the individual components below.

Stress detection: We build novel methods and models for detecting stress. We demonstrate the applicability of our methods across different devices (commodity, clinical-grade, and custom-made sensors), studies, demographics, and participants. We further create models to incorporate the context of a user along with physiological signals to better detect stress in-the-wild. We intend to release the various stress detection models so that they can be used and improved by other researchers.

Receptivity towards interventions: We explore participant receptivity towards actual behavioral change interventions and report novel and significant results, e.g., being more receptive to interventions leads to higher goal completion likelihood. We further build offline, online, and personalized machine learning models to detect the state-of-receptivity towards interventions and validate our model with independent studies to show the generalizability of our models. We intend to release the models for detecting receptivity to further advance the research in state-of-receptivity.

Modality for intervention delivery: We plan to explore how the choice of device for intervention receptivity changes with context, and hope to build novel algorithms to best determine the choice of device for delivering the interventions. 


\section{BIOGRAPHICAL SKETCH}

I am a Ph.D. candidate in the Department of Computer Science at Dartmouth College, working with Prof. David Kotz (Champion International Professor, Department of Computer Science, Dartmouth College). I started my Ph.D. in the fall of 2015. I will complete my thesis proposal in the summer of 2019 and plan to defend my thesis by spring 2021. Given the timeline for my Ph.D. defense, participating in the doctoral colloquium at UbiComp 2019 will give me the opportunity to receive feedback on my on-going work and can help shape my future thesis plans and direction.

\section{REFERENCES}

[1] Begum Egilmez, Emirhan Poyraz, Wenting Zhou, Gokhan Memik, Peter Dinda, and Nabil Alshurafa. 2017. UStress: Understanding college student subjective stress using wrist-based passive sensing. In IEEE International Conference on Pervasive Computing and Communications Workshops (PerCom Workshops). IEEE, 673-678. https://doi.org/10. 1109/PERCOMW.2017.7917644

[2] Andreas Filler, Tobias Kowatsch, Severin Haug, Fabian Wahle, Thorsten Staake, and Elgar Fleisch. 2015. MobileCoach: A novel open source platform for the design of evidence-based, scalable and low-cost behavioral health interventions: overview and preliminary evaluation in the public health context. In Wireless Telecommunications Symposium (WTS), 2015. IEEE, 1-6.

[3] Martin Gjoreski, Mitja Luštrek, Matjaž Gams, and Hristijan Gjoreski. 2017. Monitoring stress with a wrist device using context. Fournal of Biomedical Informatics 73 (Sep 2017), 159-170. https://doi.org/10.1016/ j.jbi.2017.08.006

[4] Jennifer A. Healey and Rosalind W. Picard. 2005. Detecting stress during real-world driving tasks using physiological sensors. Intelligent Transportation Systems, IEEE Transactions on 6, 2 (2005), 156-166.

[5] Karen Hovsepian, Mustafa Al'Absi, Emre Ertin, Thomas Kamarck, Motohiro Nakajima, and Santosh Kumar. 2015. cStress: Towards a Gold Standard for Continuous Stress Assessment in the Mobile Environment. Proceedings of the ACM International foint Conference on Pervasive and Ubiquitous Computing (UbiComp) (2015), 493-504. https://doi.org/10. $1145 / 2750858.2807526$

[6] Jan-Niklas Kramer, Florian Künzler, Varun Mishra, Bastien Presset, David Kotz, Shawna Smith, Urte Scholz, and Tobias Kowatsch. 2018. Investigating Intervention Components and Exploring States of Receptivity for a Smartphone App to Promote Physical Activity: Study Protocol of the ALLY Micro-Randomized Trial. FMIR Research Protocols, forthcoming (2018).

[7] Abhinav Mehrotra, Robert Hendley, and Mirco Musolesi. 2019. NotifyMeHere: Intelligent Notification Delivery in Multi-Device Environments. In Proceedings of the 2019 Conference on Human Information Interaction and Retrieval (CHIIR '19). ACM, New York, NY, USA, 103111. https://doi.org/10.1145/3295750.3298932

[8] Abhinav Mehrotra, Mirco Musolesi, Robert Hendley, and Veljko Pejovic. 2015. Designing content-driven intelligent notification mechanisms for mobile applications. In Proceedings of the ACM International Joint Conference on Pervasive and Ubiquitous Computing. ACM, 813824.

[9] Abhinav Mehrotra, Veljko Pejovic, Jo Vermeulen, Robert Hendley, and Mirco Musolesi. 2016. My phone and me: understanding people's receptivity to mobile notifications. In Proceedings of the SIGCHI Conference on Human Factors in Computing Systems (CHI). ACM, 1021-1032.
[10] Varun Mishra, Tian Hao, Si Sun, Kimberly N. Walter, Marion J. Ball, Ching-Hua Chen, and Xinxin Zhu. 2018. Investigating the Role of Context in Perceived Stress Detection in the Wild. In Proceedings of the 2018 ACM International foint Conference and 2018 International Symposium on Pervasive and Ubiquitous Computing and Wearable Computers - UbiComp '18. ACM Press, New York, New York, USA, 1708-1716. https://doi.org/10.1145/3267305.3267537

[11] Varun Mishra, Gunnar Pope, Sarah Lord, Stephanie Lewia, Byron Lowens, Kelly Caine, Sougata Sen, Ryan Halter, and David Kotz. 2018. The Case for a Commodity Hardware Solution for Stress Detection. In Proceedings of the ACM International foint Conference on Pervasive and Ubiquitous Computing Adjunct (UbiComp'18). ACM. https://doi.org/10. $1145 / 3267305.3267538$

[12] Leanne G Morrison, Charlie Hargood, Veljko Pejovic, Adam WA Geraghty, Scott Lloyd, Natalie Goodman, Danius T Michaelides, Anna Weston, Mirco Musolesi, Mark J Weal, et al. 2017. The effect of timing and frequency of push notifications on usage of a smartphone-based stress management intervention: An exploratory trial. PloS one 12, 1 (2017), e0169162.

[13] Amir Muaremi, Agon Bexheti, Franz Gravenhorst, Bert Arnrich, and Gerhard Troster. 2014. Monitoring the impact of stress on the sleep patterns of pilgrims using wearable sensors. In IEEE-EMBS International Conference on Biomedical and Health Informatics (BHI). IEEE, 185-188. https://doi.org/10.1109/BHI.2014.6864335

[14] Inbal Nahum-Shani, Eric B Hekler, and Donna Spruijt-Metz. 2015. Building health behavior models to guide the development of just-intime adaptive interventions: A pragmatic framework. Health Psychology 34, S (2015), 1209.

[15] Inbal Nahum-Shani, Shawna N Smith, Bonnie J Spring, Linda M Collins, Katie Witkiewitz, Ambuj Tewari, and Susan A Murphy. 2016. Just-intime adaptive interventions (JITAIs) in mobile health: key components and design principles for ongoing health behavior support. Annals of Behavioral Medicine 52, 6 (2016), 446-462.

[16] Mikio Obuchi, Wataru Sasaki, Tadashi Okoshi, Jin Nakazawa, and Hideyuki Tokuda. 2016. Investigating interruptibility at activity breakpoints using smartphone activity recognition API. In Proceedings of the 2016 ACM International foint Conference on Pervasive and Ubiquitous Computing: Adjunct. ACM, 1602-1607.

[17] Martin Pielot, Tilman Dingler, Jose San Pedro, and Nuria Oliver. 2015. When attention is not scarce-detecting boredom from mobile phone usage. In Proceedings of the ACM International Joint Conference on Pervasive and Ubiquitous Computing. ACM, 825-836.

[18] R. Rosmond and P. Björntorp. 1998. Endocrine and metabolic aberrations in men with abdominal obesity in relation to anxio-depressive infirmity. Metabolism 47, 10 (1998), 1187-1193.

[19] Hillol Sarker, Moushumi Sharmin, Amin Ahsan Ali, Md Mahbubur Rahman, Rummana Bari, Syed Monowar Hossain, and Santosh Kumar. 2014. Assessing the availability of users to engage in just-in-time intervention in the natural environment. In Proceedings of the ACM International foint Conference on Pervasive and Ubiquitous Computing. ACM, 909-920.

[20] Philip Schmidt, Attila Reiss, Robert Duerichen, Claus Marberger, and Kristof Van Laerhoven. 2018. Introducing WESAD, a Multimodal Dataset for Wearable Stress and Affect Detection. In Proceedings of the 20th ACM International Conference on Multimodal Interaction (ICMI '18). ACM, New York, NY, USA, 400-408. https://doi.org/10.1145/3242969. 3242985 